\title{
Simultaneous imaging of neural activity in 3D (Presentation Video)
}

\section{Rafael Yuste}

Rafael Yuste M.D., "Simultaneous imaging of neural activity in 3D (Presentation Video)," Proc. SPIE 9305, Optical Techniques in Neurosurgery, Neurophotonics, and Optogenetics II, 93053C (21 April 2015); doi: $10.1117 / 12.2197212$

SPIE. Event: SPIE BiOS, 2015, San Francisco, California, United States 


\title{
Simultaneous imaging of neural activity in 3D (Presentation Video)
}

\author{
Rafael Yuste, Columbia University (USA)
}

\begin{abstract}
Many people suffering from diseases such as epilepsy, autism, or are diagnosed with schizophrenia, Alzheimer's, or Parkinson's disease that cannot be helped because we do not understand how the brain works, notes Rafael Yuste in this BiOS Hot Topic talk. So scientists are working on methodologies to study the brain, visualize neurons, and map the connections, in order to comprehend neural circuitry in its entirety and develop treatments. Neuroscientists are currently able to visualize neuron activity using calcium imaging, based on changes in fluorescence intensity or spectral properties of a dye that is sensitive to fluctuations in intracellular calcium concentrations, which is directly related with neuron activity. This method works in live animals, using a window in their skull. Nonlinear microscopy provides the ability to image deeper inside the brain. The Yuste group is now working on expanding this technique to fast 3D imaging in the brains of living animals. Until not long ago, 3D visualization was only achievable by sequentially scanning different focal planes, which is very time-consuming. The implementation of holographic methods in their microscope can bring a solution.
\end{abstract}

View presentation video on SPIE's Digital Library: http://dx.doi.org/10.1117/12.2197212.4093523005001

Optical Techniques in Neurosurgery, Neurophotonics, and Optogenetics II, edited by Henry Hirschberg, Steen J. Madsen, E. Duco Jansen, Qingming Luo, Samarendra K. Mohanty, Nitish V. Thakor, Proc. of SPIE Vol. 9305 ,

93053C · C 2015 SPIE · CCC code: 1605-7422/15/\$18 · doi: 10.1117/12.2197212 